\title{
Fate of (D-Ala2)-deltorphin-I-like immunoreactive neurons in 6-hydroxydopamine lesioned rat brain
}

\author{
A. Casini, ${ }^{1}$ A. Pinna, ${ }^{2}$ I. Tooyama, ${ }^{3}$ H. Kimura, ${ }^{3}$ G. Di Chiara, ${ }^{2}$ T.G. Renda ${ }^{1}$ \\ ${ }^{1}$ Department of Human Anatomy, University "La Sapienza", Rome, Italy; ${ }^{2}$ Department of Toxicology, \\ University of Cagliari, Italy; ${ }^{3}$ Molecular Neuroscience Research Center, Shiga University of Medical \\ Science, Otsu, Japan
}

(c)2004, European Journal of Histochemistry

The use of a polyclonal antiserum specific to C-terminal tetrapeptide amide of (D-Ala ${ }^{2}$ )deltorphin-I, a naturally occurring amphibian skin opioid peptide, has already demonstrated the presence of immunoreactive neurons in rat midbrain. Double immunostaining identified these neurons as a subpopulation of the mesencephalic dopaminergic neurons that were also tyrosine hydroxylase-immunopositive and calbindin-D28kD- negative, namely, the neurons predominantly affected in Parkinson disease. We followed the fate of these neurons after a monolateral injection of 6-hydroxy-dopamine into rat brain. Almost all the immunopositive neurons and their nigrostriatal, mesolimbic and mesocortical projections on the side ipsilateral to the lesion disappeared. Only a few scattered immunopositive neurons within the substantia nigra, pars compacta, and those of supramammillary nucleus remained unaffected. The consistent overlap of dopamine and this new molecule provides a further key to identifying the mammalian counterpart of these amphibian skin opioid peptides.

Key words: Amphibian skin peptides; Opioid peptides; Deltaopioid receptors; Dopaminergic system; Tyrosine hydroxylase; Calbindin-D28kD, Parkinson disease.

Correspondence: Prof. Tindaro G. Renda, Department of Human Anatomy, via Alfonso Borelli 50, 00161 Rome, Italy. Tel. +39.06.490125; Fax +39.06.4452349.

E-mail: tindaro.renda@uniroma1.it

Paper accepted on January 21, 2004

European Journal of Histochemistry 2004; vol. 48 issue 2 [Apr-Jun]: 135-140
( D -Ala ${ }^{2}$ )deltorphin-I (DADTI), is a heptapeptide amide (Tyr-D-Ala-Phe-Asp-Val-Val-Gly. $\mathrm{NH}_{2}$ ) originally extracted, together with its analogue (DAla ${ }^{2}$ )-deltorphin II (Tyr-D-Ala-Phe-Glu-Val-ValGly. $\mathrm{NH}_{2}$ ), from the skin of the South American frog Phyllomedusa bicolor (references: Erspamer, 1992). Together with dermorphins they constitute a family of heptapeptide amides whose main biochemical characteristic is the presence in position 2 of a D-amino acid that seems essential to their biological action. They display high affinity and selectivity for the opioid receptors: dermorphins for mu opioid receptors and deltorphins for delta opioid receptors (Dupin et al., 1991; Gouarderes et al., 1993; Renda et al., 1993). In pharmacological studies in the rat, the intracerebroventricular injection of DADTI or DADTII induces locomotory stereotypies (e.g. grooming, sniffing and rearing) mediated by the dopamine release and D1 receptor activation at basal ganglia level and in particular within the nucleus accumbens (Longoni et al., 1991). It also increases social contacts (Negri et al., 1991) and, in mice, improves memory consolidation (Pavone et al., 1990). Two different cDNAs, one coding for dermorphins and the other for deltorphins, have been isolated from the skin of Phyllomedusa sauvagei and Phyllomedusa bicolor (Richter et al., 1987, 1990). Studies seeking a mammalian counterpart of amphibian opioid peptides have yielded promising information on dermorphins (Buffa et al., 1982; Tsou et al., 1985; Mor et al., 1990), but fewer data on deltorphins. To fill this gap in knowledge our group has produced a polyclonal antiserum that specifically recognizes the C-terminal tetrapeptide amide of DADTI (Asp-Val-Val-Gly. $\mathrm{NH}_{2}$ ) (Abe et al., 1992). Throughout this paper we will indicate all the structures recognized by this antiserum as DADTIimmunoreactive. DADTI-immunoreactive neurons were detected in discrete brain areas partially overlapping with the mesencephalic dopaminergic system $(A 8, A 9$ and $A 10)$ in the adult mouse and rat 
brain (Abe et al., 1992; Tooyama et al., 1993; Renda et al., 1997).

Double-immunostaining procedures demonstrated that DADTI-immunoreactive neurons represent about one third of the whole tyrosine hydroxylase ( $\mathrm{TH}$ )-immunoreactive neurons present in the mesencephalic dopaminergic area (Tooyama et al., 1993; Renda et al., 1997). Because these neurons are also immunonegative for Calbindin D28kD (Tooyama et al., 1993) they are considered the dopaminergic neuron subpopulation most directly involved in Parkinson disease lesions (Yamada et al., 1990). The distribution patterns of their immunopositive terminals corresponds to a part of the nigrostriatal, mesolimbic, mesocortical and mesothalamic pathways normally projecting from these mesencephalic areas. The same antiserum was also used to study the distribution patterns of immunoreactive cells during the first three weeks after birth in rat brain (Nonomura et al., 1994), during the brain ontogeny in rat embryos (Park et al., 2000), and in various other apparatus (Abe et al., 1994; Fujimiya et al., 1994; Matsui J. et al., 1994; Matsui S. et al., 1994; Sunday et al., 2001). All these data suggest that mesencephalic dopamine and this partly identified molecule that shares with DDTI the epitope recognized by our antiserum have close neuroanatomical and functional relationships.

To elucidate these relationships further, in this study we followed the fate of the DADTI-immunoreactive neurons after the monolateral chemical lesion of the dopaminergic system caused by 6-hydroxydopamine (6-OHDA), a selective dopaminergic monoamine neurotoxin currently used to create models of hemiparkinsonian rats.

\section{Materials and Methods Animals}

Eight male rats (Sprague-Dawley, Charles, River, Calco, Italy), weighing 275-300 g, were injected with 6-0HDA on the left brain side as previously described (Morelli et al., 1993). In brief, the rats, under chloral hydrate anesthesia (400 mg/kg i.p.), received an injection of $6-0 \mathrm{HDA}-\mathrm{HCl}$ (Sigma Chem., St Louis, USA: $8 \mu \mathrm{g}$ in $4 \mu \mathrm{L}$ of saline containing $0.05 \%$ ascorbic acid) at left medial forebrain bundle level (coordinates A -2.2, L 1.5, V 7.8, according to the atlas of Pellegrino et al., 1979). A second group of eight control rats was sham-operated and received the vehicle alone. All procedures were approved by the local Ethical Committee and met the NIH guidelines for animal care. Ten days after the injection the rats were tested for contralateral rotational behavior by $0.3 \mathrm{mg} / \mathrm{kg}$ apomorphine. We selected only the animals exhibiting more than 100 contralateral turns/ 1 hour, because a published report (Hefti et al., 1980) and our experience showed that they could be considered as more than $95 \%$ dopamine depleted on the lesioned side.

\section{Tissue preparation}

On day 15 after the injection, successfully lesioned rats and control rats, under anesthesia, were perfused with cold saline and then with cold fixative (PAF) containing 4\% paraformaldehyde, $0.2 \%$ picric acid in $0.1 \mathrm{M}$ phosphate buffer, $\mathrm{pH} 7.4$ (PB), with $0.35 \%$ glutaraldehyde added. The brains were quickly removed and postfixed for $48 \mathrm{~h}$ with cold PAF alone, and then stored at $4^{\circ} \mathrm{C}$ in PB containing $15 \%$ sucrose till use. Brain specimens were frozen and cut in a cryostat into $30 \mu \mathrm{m}$-thick coronal sections, which were collected in cold buffered 15\% sucrose. Before immunohistochemical procedures, to improve tissue permeability, free-floating sections were incubated for at least 3 days in saline PB containing $0.3 \%$ Triton $\mathrm{X}-100$ (PBST) at $4{ }^{\circ} \mathrm{C}$.

\section{Immunohistochemistry}

To inactivate the endogenous peroxidase activity, the sections were pre-treated for $30 \mathrm{~min}$ at room temperature with PBST containing $0.1 \%$ sodium azide and $0.5 \% \mathrm{H}_{2} \mathrm{O}_{2}$. To improve the quality of immunostaining, sections were then submitted to light proteolysis by incubation for $10 \mathrm{~min}$ at $37^{\circ} \mathrm{C}$ in PBST containing 1 U.I./mL papain (Mercuripapain, Sigma, St.Louis, USA). The proteolytic activity was then stopped by directly transferring the sections into 4\% buffered paraformaldehyde for 20 min at room temperature followed by a thorough washout with PBST $(3 \times 10 \mathrm{~min})$. Sections were then incubated for 3 days at $4^{\circ} \mathrm{C}$ with our polyclonal antiserum, recognizing the $\mathrm{C}$-terminal tetrapeptide amide of DADTI, diluted $1: 10,000$ with PBST containing $0.5 \%$ bovine serum albumin (BSA, Sigma). The sections were then incubated for $2 \mathrm{~h}$ at room temperature with biotinylated goat anti-rabbit IgG (diluted 1:2000; Vector Laboratories, Burlingame, CA, USA) and for $1 \mathrm{~h}$ at room temperature with streptavidin-peroxidase complex (diluted 1: 2,000, $A B C$ Elite, Vector). After each step, reagents were diluted and sections were rinsed with PBST. The peroxidase activity was visualized by reaction for $3 \mathrm{~min}$ 
at room temperature with a solution containing $0.04 \%$ 3-3' diaminobenzidine tetrahydrochloride (DAB, Fluka, Buchs, Switzerland), 0.4\% nickel ammonium sulphate and $0.003 \% \mathrm{H}_{2} \mathrm{O}_{2}$ in $0.05 \mathrm{M}$ Tris- $\mathrm{HCl}$ buffer, $\mathrm{pH} 7.6$ giving a dark blue granular precipitate. The stained sections were mounted on glass slides, dehydrated, cleared and coverslipped with Permount (Fisher Scientific, USA). The characteristics of the DADTI antiserum have been described elsewhere (Abe et al., 1992, 1994; Tooyama et al., 1993). In brief, the synthetic heptapeptide amide DADTI (Sigma) conjugated with polyglutamate was used as antigen. The antiserum was characterized and the epitope (Asp-Val-ValGly. $\mathrm{NH}_{2}$ ) established in spot dot tests and blocking studies against several DADTI analogs and fragments and several biologically active known peptides (a complete list in Abe et al., 1992, 1994). For control experiments, the primary DADTI antiserum was substituted with buffer or preimmune rabbit serum, or with preabsorbed antiserum (preincubation overnight at $4^{\circ} \mathrm{C}$ with synthetic DADTI (Sigma) $25 \mu \mathrm{g} / \mathrm{mL}$ antiserum at working dilution). None of the control sections showed positive staining. By contrast, the addition of several other biologically active peptides, including dermorphin, met- and leuenkephalin and dynorphin (Sigma) (up to 100 $\mu \mathrm{g} / \mathrm{mL}$ antiserum at the working dilution) left immunostaining unchanged.

To assess the relationships of the DADTIimmunoreactive neurons with the dopaminergic system, several sections previously immunostained for DADTI were double immunostained by a further 3day incubation at $4^{\circ} \mathrm{C}$ with a monoclonal anti-tyrosine hydroxylase ( $\mathrm{TH}$, Chemicon International Inc., Temicula, CA, USA) (raised in mouse) diluted 1:5000, followed by a biotinylated goat anti-mouse, rat adsorbed, IgG (Vector) diluted 1:2000 and $A B C$-peroxidase complex. The peroxidase activity was then revealed by the Novared kit (Vector), producing a red precipitate. Controls were made by omitting the secondary antiserum in the double immunostaining procedure.

To control the effectiveness of 6-OHDA injection, some randomly chosen sections both at forebrain and at midbrain levels of lesioned and sham-operated rats were immunostained with $\mathrm{TH}$ monoclonal antiserum alone.

DADTI-immunoreactive neurons were counted using a dedicated image analysis software (I.A.S., Delta Sistemi, Rome, Italy), on at least 6 consecu- tive coronal sections at comparable midbrain levels, containing substantia nigra and ventral tegmental area, from five successfully lesioned brain specimens. Values are expressed as mean \pm SD of DADTIimmunoreactive neurons per each side of coronal sections. Any further statistical or densitometric evaluation was retained superfluous because values distinctly differed in lesioned sides vs contralateral non-lesioned sides.

\section{Results}

According to the foregoing criteria, in seven of the eight treated rats, injection of 6-OH-DA successfully lesioned the ipsilateral dopaminergic system around the injection site. The 6-OH-DA-induced lesions were clearly visible in sections immunostained with $\mathrm{TH}$ antiserum. Ipsilaterally to the lesion, $\mathrm{TH}$-immunopositive nerve cell bodies and fibers were almost completely lost within the substantia nigra (A9) and to a lesser extent in the neighboring ventral tegmental area (A10) (data not shown). Wide forebrain areas, including caudate-putamen, nucleus accumbens, septum and cingulate cortex, towards which these nuclei normally project their fibers, were almost completely emptied (data not shown). In the same mesencephalic areas, DADTIimmunoreactive neurons almost disappeared on the side ipsilateral to the lesion (Figure 1a). Only a few DADTI-immunoreactive, but TH-negative, neurons lay scattered in these mesencephalic areas especially in the substantia nigra, pars compacta (Figure 1c). Double immunostaining also confirmed the features of the co-localization with TH both in the contralateral side of 6-OH-DA-injected animals (Figure Ib) and in normal controls, as already reported in previous investigations (Tooyama et al., 1993; Renda et al., 1997). Several attempts to characterize better the few DADTI-positive/TH-negative neurons spared by the 6-OH-DA injury added no further information. DADTI-immunoreactive neuron counts were markedly lower on the side ipsilateral than on the side contralateral to the lesion (average count $4.033 \pm 0.52$ SEM, range $0-8$, vs $168.1 \pm 3.66$, range 135-200 per coronal section) (Figure 2).

Ipsilaterally to the lesion, DADTI-immunoreactive nerve fibers were also dramatically reduced in the same forebrain areas, including dorsolateral caudate-putamen (Figure 3b), nucleus accumbens shell (Figure $3 c$, e), lateral septum, medial forebrain bundle, olfactory tubercle and cingulate cortex (data not shown). Contralaterally to the lesion, they 


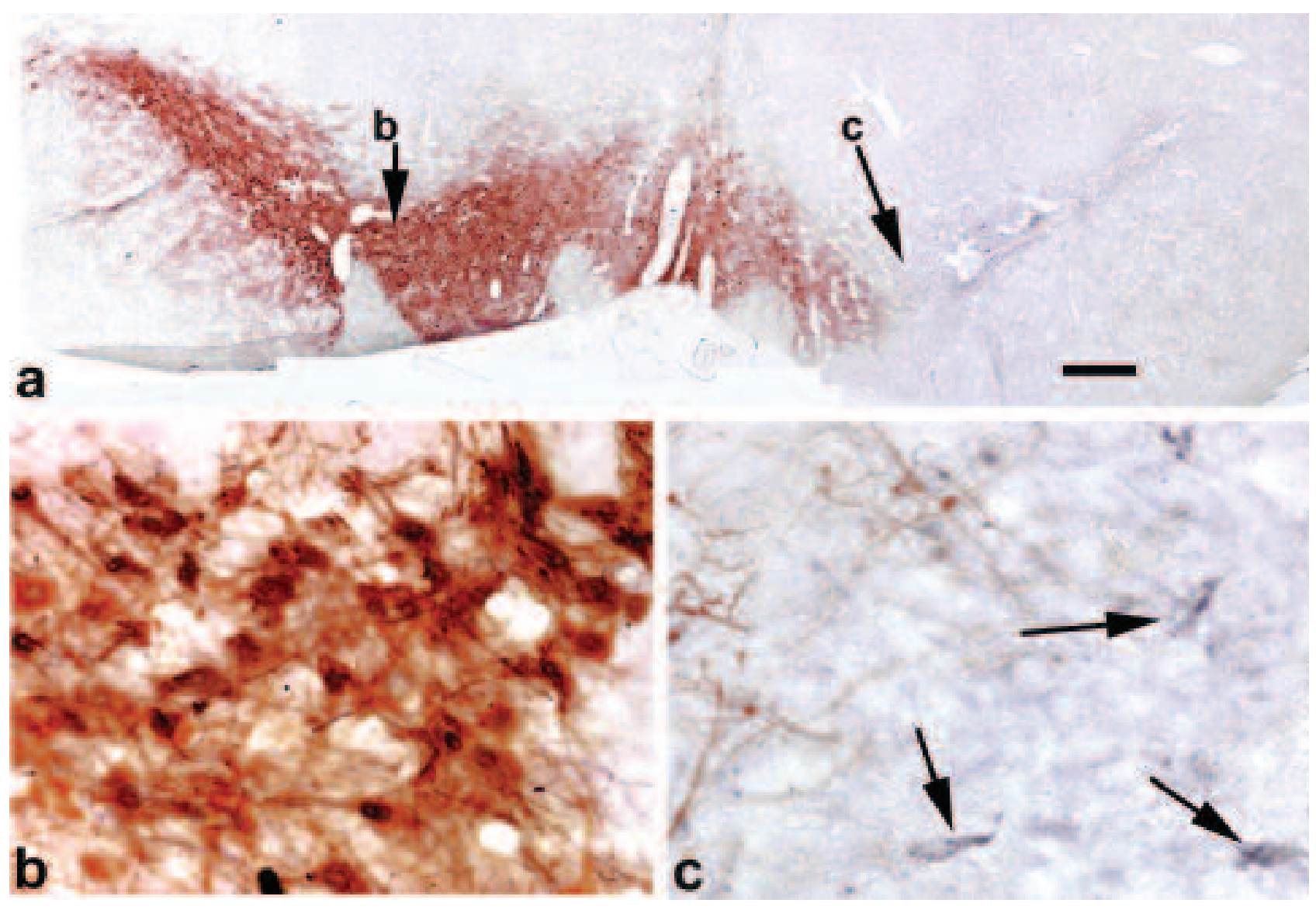

Figure 1. a. Computer aided merge of a midbrain coronal section double immunostained for DADTI (blue) and TH (red). Arrows indicate the sites reported at higher magnification in figures $b$ and $c$. Several double immunostained neurons and a conspicuous nerve fiber network occupy the A9 area contralateral to the lesioned side (b), whereas only a few DADTI-immunoreactive and TH-negative neurons are clearly shown ipsilaterally (c, arrows) together with a few TH-immunoreactive nerve fibers. Scale bar: $a=700 \mu \mathrm{m} ; b, c=30 \mu \mathrm{m}$.

were found in all these regions and had the same distribution and staining intensity as they did in controls (Figure 3a, c, d). Finally, no side differences were visible at the level of supramammillary nucleus (SM) or in the main and accessory olfactory bulbs, where DADTI-immunoreactive and THimmunonegative neurons and fibers were found, as shown in previous studies (Tooyama et al., 1993; Renda et al., 1997) (data not shown).

\section{Discussion}

When we lesioned the rat dopaminergic system by injecting 6-OH-DA, the DADTI-immunoreactive neurons almost completely disappeared in the midbrain areas A9 and partially disappeared in Al0. These findings definitively confirm previous indications cumulated during the past decade by our group using the specific antiserum that recognizes the C-terminal tetrapeptide amide of DADTI (Abe et al., 1992; Tooyama et al., 1993; Nonomura et al.,
1994; Renda et al., 1997; Park et al., 2000). Previous evidence suggesting close relationships between these neurons and the dopaminergic system came only from double immunostaining procedures. With these techniques we identified a subpopulation of dopaminergic mesencephalic neurons notably present within the ventral tier of the substantia nigra, pars compacta and ventral tegmental area (Tooyama et al., 1993; Renda et al., 1997). These neurons constitute about one third of the whole THimmunoreactive neuronal population present in that area and nearly all of them (97.8\%) co-localized TH. Yet remarkably few $(7.7 \%)$ co-localized calbindin-D28kD (Tooyama et al., 1993). Hence this subpopulation closely corresponds to the dopaminergic neurons thought to be most directly and strongly affected by Parkinson disease lesions (Yamada et al., 1990). These findings also clearly corroborate another key point we have already stressed in previous reports (Tooyama et al., 1993; Renda et al., 1997). The lesioning allowed us to 


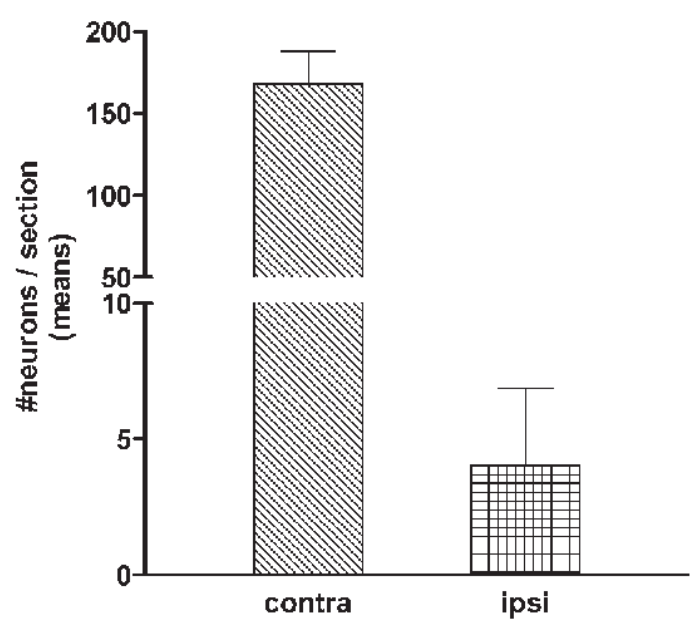

Figure 2. DADTI-immunoreactive neuron counts made on several coronal sections at midbrain level contralaterally (contra) and ipsilaterally (ipsi) to the lesioned side and expressed as mean number per coronal section.

detect, especially in the lesioned side, a small number of DADTI-immunoreactive neurons (about 1$2 \%$ ) not displaying $\mathrm{TH}$-immunoreactivity that normally lay among the DADTI- and TH-immunoreactive neurons within areas $A 9$ and $A 10$. We also found DADTI-immunoreactive, but TH-negative, unaffected neurons on both brain sides in nondopaminergic areas (e.g. supramammillary nucleus, main and accessory olfactory bulbs). This population had the same features we described in previous investigations (Tooyama et al., 1993; Renda et al., 1997).

Because a cDNA identical to that isolated from amphibian skin has still to be found in mammalian tissues, we cannot fully identify the DADTIimmunoreactive molecule recognized by our antiserum. All the results so far cumulated nonetheless imply that our antiserum recognizes a new molecule that shares structural and functional homologies with DADTI. All data collected to date clearly indicate that this molecule has its own identity. Despite being found mainly within a subpopulation of dopaminergic neurons in the rat brain, it is also present in other systems (Abe et al., 1994; Fujimiya et al., 1994; Matsui J. et al., 1994; Matsui S. et al., 1994; Sunday et al., 2001). It is present in varying amounts in various tissues and at different ages. Previous studies conducted in rat embryos (Park et al., 2000) and newborns (Nonomura et al., 1994) detected DADTI-immunoreactive neurons in widespread brain sites, whereas in the adult they became

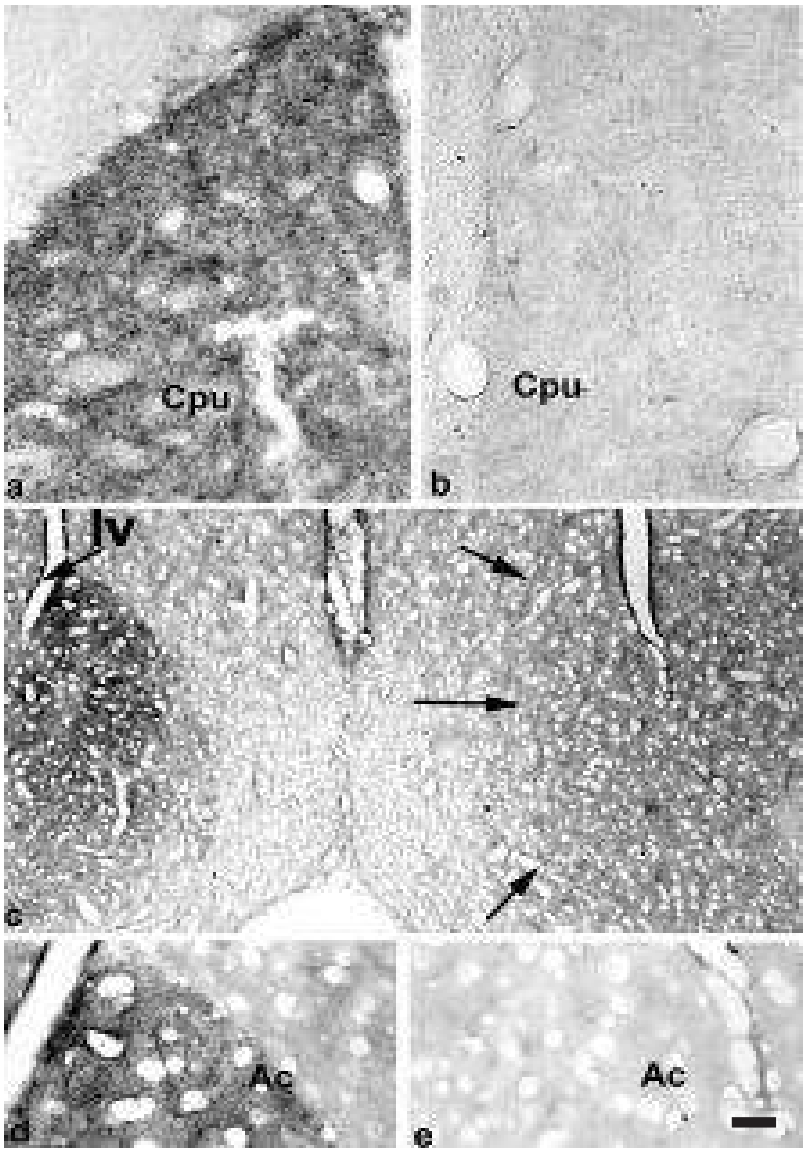

$\overline{\text { Figure 3. Cryostatic sections of a lesioned rat brain, at various }}$ coronal levels, immunostained for DADTI. a-b. Detail of the dorsolateral region of caudate putamen $(\mathrm{CPu})$ filled with numerous DADTI-immunoreactive nerve fibers contralaterally (a) but almost completely empty on the side ipsilateral (b) to the lesion. c. Computer-aided merge to compare the two sides of the forebrain region at nucleus accumbens level. Contralateral and ipsilateral sides are reported at higher magnification in $\mathbf{d}$ and $\mathbf{e}$, respectively. DADTI-immunoreactive nerve fibers mainly fill the nucleus accumbens shell (Ac) contralaterally (d) and are almost absent ipsilaterally to the lesioned side (arrows) (e). Iv = lateral ventricle. Scale bar: $c=2.25 \mathrm{~mm}$; $a-b, d-e=65 \mu \mathrm{m}$.

far less numerous and were mainly confined within the dopaminergic areas. Also in other systems, including gut (Fujimiya et al., 1994) and lung (Matsui S. et al., 1994; Sunday et al., 2001) DADTI-immunoreactive cells have been shown mainly in the embryonic and perinatal ages. Whether this mammalian DADTI-like molecule is an opioid-like molecule remains unclear. Two lines of evidence suggest that it is. First, the sites where DADTI-immunoreactive nerve fibers reach their major concentration in mouse and rat brain impressively correspond to the main sites of $\delta$-opioid receptors as identified using deltorphins as ligands (Dupin et al., 1991; Gouarderes et al., 1993; Renda et al., 1993). Second, in mouse fetal lung, our anti- 
serum detected DADTI-immunoreactive alveolar cells that express $\delta$ - and $\mu$-opioid receptors and dose-dependently display opposite effects to deltorphin and dermorphin treatment in vitro (Sunday et al., 2001). Our antiserum also immunostained neurons from rat substantia nigra cultured in vitro ( $\mathrm{Yu}$, personal communication) as did a new monoclonal antiserum, specific for $\mathrm{N}$-terminal portion of DADTI (Yu et al., 2000). Overall, these data intriguingly indicate that during the early stages of life the DADTI-like molecule may intervene in morphogenetic or differentiation processes or both. In the adult, its presence diminishes and is almost restricted to the dopaminergic neurons. Interestingly, treatment with a $\delta$-opioid receptor agonist significantly improves dopaminergic cell viability in vitro, and clearly protects in vivo against 6-0HDA-induced dopamine depletion (Borlongan et al., 2000). The cohabitation of an opioid peptide and dopamine within the same neuron could, therefore, assume a new intriguing meaning, possibly providing new clues to the pathogenesis of Parkinson's disease. Hence, these findings warrant increased efforts to gain full information on the DADTI-like molecule recognized by our antiserum in mammalian tissues.

\section{Acknowledgments}

This work was supported by a research grant from the Italian Ministry of University (MURST), cofin 1999, grant\# 9905042854_005; and 60\%, 2001 (T.G.R.).

\section{References}

Abe H, Tooyama I, Renda T, Erspamer V, Kimura H. Production of antiserum to [D-Ala2]-deltorphin-I and its immunohistochemical application to the mouse brain. Neuroreport 1992; 3:669-72.

Abe H, Tooyama I, Renda T, Erspamer V, Kimura H. Immunohistochemical demonstration of [D-Ala2]-deltorphin-I in amacrine cells of rat retina. Peptides 1994; 15:49-54.

Borlongan CV, Su TP, Wang Y. Treatment with delta opioid peptide enhances in vitro and in vivo survival of rat dopaminergic neurons. Neuroreport 2000; 11:923-26.

Buffa R, Solcia E, Magnoni E, Rindi G, Negri L, Melchiorri P. Immunohistochemical demonstration of a dermorphin-like peptide in the rat brain. Histochemistry 1982; 76:273-76.

Dupin S, Tafani A, Mazarguil H, Zaiac JM. (125I)-(D-Ala2)-deltorphin-I: a high affinity, delta selective opioid receptor ligand. Peptides $1991 ; 12: 825-30$

Erspamer $\mathrm{V}$. The opioid peptides of the amphibian skin. Int $\mathrm{J}$ Dev Neurosci 1992; 10:3-30.

Fujimiya M, Okumiya K, Renda TG, Kimura H, Maeda T. Demonstration of (D-Ala2)-deltorphin I-like immunoreactivity in mucosal epithelial cells of the rat gastrointestinal tract. Peptides 1994; 15: 1095-100.

Gouarderes C, Tellez S, Tafani JA, Zajac JM. Quantitative autoradio- graphic mapping of delta-opioid receptors in the rat central nervous system using [ $\left.{ }^{125} \mathrm{I}\right][\mathrm{D}-\mathrm{Ala} 2]-$ deltorphin-I. Synapse 1993; 13:231-40.

Hefti F, Melamed E, Sahakian BJ, Wurtman RJ. Circling behavior in rats with partial, unilateral nigro-striatal lesions: effect of amphetamine, apomorphine and dopa. Pharmacol Biochem Behav 1980; 12: 185-88.

Longoni R, Spina L, Mulas A, Carboni E, Garau L, Melchiorri P, Di Chiara G. (D-Ala2)-deltorphin-II: d1-dependent stereotypies and stimulation of dopamine release in the nucleus accumbens. J Neurosci 1991; 11:1565-76.

Matsui J, Fujimiya M, Matsui S, Amakata Y, Renda TG, Kimura H, Maeda T. Transient expression of (D-Ala2)-deltorphin I-like immunoreactivity in prenatal rat small intestine. $\mathrm{J}$ Histochem Cytochem 1994; 42:1377-82.

Matsui S, Fujimiya M, Matsui J, Amakata Y, Renda TG, Kimura H, Maeda T. Localization of (D-Ala2)-deltorphin I-like immunoreactivity in perinatal rat respiratory system. Histochemical J 1994; 26 : 648-54.

Mor A, Pradelles P, Delfour A, Montagne JJ, Quintero FL, Conrath M, $\mathrm{Nicolas} \mathrm{P}$. Evidence for pro-dermorphin processing products in rat tissues. Biochem Biophys Res Comm 1990; 170:30-8.

Morelli M, Fenu S, Pinna A, Cozzolino A, Carta A, Di Chiara G. "Priming" to dopamine agonist-induced contralateral turning as a model of non-associative sensitization to the expression of the postsynaptic dopamine message. Behav Pharmacol 1993; 4:389-97.

Negri L, Noviello $V$, Angelucci F. Behavioural effects of deltorphins in rats. Eur J Pharmacol 1991 ; 209:163-8.

Nonomura K, Tooyama I, Abe H, Renda T, Erspamer V, Shimada M, Kimura H. Postnatal development of (D-Ala2)-deltorphin-I-like immunoreactive structures in the rat brain. J Comp Neurol 1994; 349:223-43.

Park M, Tokunaga Y, Kimura H, Tooyama I, Maeda T, Renda T. Ontogeny of (D-Ala2)-deltorphin I-like immunoreactive neurones in foetal rat brain. J Chem Neuroanat 2000;18:11-22.

Pavone F, Populin R, Castellano C, Kreil G, Melchiorri P. Deltorphin, a naturally occurring peptide with high selectivity for delta opioid receptors, improves memory consolidation in two inbred strains of mice. Peptides 1990; 11:591-94.

Pellegrino LJ, Pellegrino AS, Cushman AJ. A stereotaxic atlas of the rat brain. Plenum Press, New York-London, 1979.

Renda T, Erspamer V, Tooyama I, Kimura H. Mesencephalic $\delta$-opioid peptidergic ascending projection with special reference to the mesolimbocortical system. In: Motta PM, ed. Recent advances in microscopy of cells, tissues and organs. Delfino Ed., Rome, 1997, pp. 239-46.

Renda T, Negri L, Tooyama I, Casu C, Melchiorri P. Autoradiographic study on (3H)-(D-Ala2)-deltorphin-I binding sites in the rat brain. Neuroreport 1993; 4:1143-46.

Richter K, Egger R, Kreil G. D-alanine in the frog skin peptide dermorphin is derived from L-alanine in the precursor. Science 1987; 238:200-2.

Richter K, Egger R, Negri L, Corsi R, Severini C, Kreil G. cDnas encoding (D-Ala2)-deltorphin precursors from skin of Phyllomedusa bicolor also contain genetic information for three dermorphin-related opioid peptides. Proc Natl Acad Sci Usa 1990; 87:4836-9.

Sunday ME, Haley KJ, Emanuel RL, Torday JS, Asokananthan N, Sikorski KA, et al. Fetal alveolar epithelial cells contain (D-Ala2)deltorphin I-like immunoreactivity. $\delta$ and $\mu$-opiate receptors mediate opposite effects in developing lung. Am J Resp Cell Mol Biol 2001; 25:447-56.

Tooyama I, Abe H, Renda T, Erspamer V, Kimura H. (D-Ala2)deltorphin I-like immunoreactivity in the adult rat brain: immunohistochemical localization. Proc Natl Acad Sci Usa 1993; 90:9635-9.

Tsou K, Wang FS, Wang SH, Tang YQ. Dermorphin-like immunoreactivity in guinea pig and rat stomach. Neuropeptides 1985; 5:449-52.

Yamada T, McGeer PL, Baimbridge KG, McGeer EG. Relative sparing in Parkinson's disease of substantia nigra dopamine neurons containing calbindin-D28kd. Brain Res 1990; 526:303-7.

Yu S, Zhao T, Fan M, Tooyama I, Kimura H, Renda TG. Production of monoclonal antibody to deltorphin-I and its immunohistochemical application to adult rat brain and cultured rat brain neurons. Peptides 2000; 21:1657-62. 